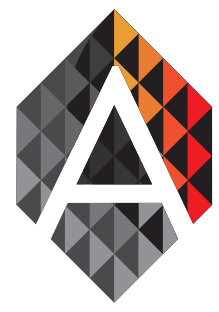

\title{
An Analysis of Software Agents, Environ-ments and Applications School (WESAAC): Retrospective, Relevance, and Trends
}

\author{
Enyo Gonçalves ${ }^{\mathrm{a}, \mathrm{d}}$, Mariela Cortés ${ }^{\mathrm{b}}$, Marcos de Oliveira ${ }^{\mathrm{a}}$, \\ Nécio Veras ${ }^{\mathrm{c}}$, Mário Falcão ${ }^{\mathrm{b}}$ and Jaelson Castro ${ }^{\mathrm{d}}$ \\ a'Universidade Federal do Ceará \\ bUniversidade Estadual do Ceará \\ 'Instituto Federal de Educação, Ciência e Tecnologia do Ceará \\ dUniversidade Federal de Pernambuco \\ enyo@ufc.br,mariela@larces.uece.br,marcos.oliveira@ufc.br, necio.veras@ifce.br, \\ mariofal-cao.es@gmail.com,jbc@cin.ufpe.br
}

\begin{tabular}{|c|c|}
\hline KEYWORD & ABSTRACT \\
\hline WESAAC; & Multi-Agent Systems (MAS) software has been increasing dramatically in last years. In \\
\hline Scoping study; & this context, the Software Agents, Environments and Applications School (WESAAC) \\
\hline Retrospective, & is a Brazilian event of MAS which is in its tenth edition. When studying a research \\
\hline Trends and & area, it is important to identify the most active groups, topics, the research trends and \\
\hline Relevance; & so forth. This study aims to investigate how the WESAAC is evolving, by analysing \\
\hline Systematic & the papers published in its ten editions. We adopted a research strategy that combines \\
\hline Mapping Stu & $\begin{array}{l}\text { scoping study and systematic review good practices. We identify the most participating } \\
\text { institutions and authors, the main topics discussed, the types of the contributions, the } \\
\text { conferences and journals that have most referenced WESAAC papers, the publications } \\
\text { with the greatest impact, and the trends in MAS. We found } 128 \text { papers over the } 10 \\
\text { WESAAC editions, which were analysed and discussed. }\end{array}$ \\
\hline
\end{tabular}

\section{Introduction}

Nowadays, agent technology has been widely applied to solve a vast set of problems. Russell and Norvig (2003) define an agent as an entity that can perceive its environment through sensors and act in the environment through actuators. According to Wooldridge (2009), agents are complex entities with behavioural properties, such as (i) autonomy (i.e., they are able to execute without interacting with humans), (ii) social ability (i.e., they are able to interact by sending and receiving messages and not by explicit task invocation, (iii) Reactivity (i.e. the capacity of perceiving the environment and respond to its changes), and (iv) Proactiveness (i.e. a goal-directed behaviour). Multi-Agent Systems (MAS) is the sub area of artificial intelligence that investigates the behaviour of a set of autonomous agents, aiming to solve a problem that is beyond the capacity of a single agent (Russel and Norvig, 2003). The agent-oriented development paradigm requires adequate techniques to explore its benefits and features to support the construction and maintenance of this type of software (Zambonelli et al., 2001).

Enyo Gonçalves, Mariela Cortés, Marcos de Oliveira, Nécio Veras, Mário Falcão and Jaelson Castro An Analysis of Software Agents, Environ-ments and Applications School (WESAAC): Retrospective, Relevance, and Trends

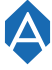

ADCAIJ: Advances in Distributed Computing and Articial Intelligence Journal Regular Issue, Vol. 6 N. 2 (2017), 19-32 elSSN: 2255-2863 - http://adcaij.usal.es (c) Ediciones Universidad de Salamanca - CC BY 
In this context, the Software Agents, Environments and Applications School (WESAAC) provides a forum for researchers, practitioners and educators to present and discuss the most recent innovations, trends, experiences and concerns in the field of software agents. The overall goal of the WESAAC is to provide an opportunity for researchers and students (both undergraduate and graduate) to discuss their aims, methods, and results of their research. The School seeks to provide students with useful guidance on various aspects of the research from established researchers and the other student attendees. It also helps participants to establish a research and social network of their peers.

Motivated by the celebration of the 10th edition of the WESAAC, this work aims to analyse the 128 full papers published in the WESAAC throughout its ten editions. This review seeks to identify the most participating institutions and authors, the main topics discussed, the types of the contributions, the conferences and journals that have most referenced WESAAC papers, the most addressed topics, the publications with the greatest impact, and the trends in MAS.

The information provided in this article may be useful in different contexts. For example, a newcomer (e.g., new research student) will be able to identify main groups, main researchers and the work already developed. Moreover, topics that have not deserved much attention by the WESAAC may be identified and become the subject of new research projects. This kind of information will also be useful for setting up possible collaborative networks.

This paper is structured as follows. In Section 2, it is described the retrospective of the event. The research method is outlined in section 3. In Section 4, it is presented the review results. In Section 5, it is discussed some threats to validity. Some related works are presented in Section 6. Finally, the conclusion and future works are presented in Section 7.

\section{Multi-Agent Systems}

Software Engineering for Multi-Agent Systems (MAS) (Weiss, 2013), (Wooldridge, 2009) has been a relevant research topic in the last 15 years, and we can now find several mature models of this approach ready to be used in a wide variety of contexts and applications. For the development of MAS, organisational models are usually employed with the objective of dealing with the freedom and autonomy that the entities, which compose this type of system, can have over decision-making during the execution of activities or actions in the context of the system. This organizational layer becomes even more necessary when we refer to open MAS (Weiss, 2013) (De Oliveira, Gonçalves and Purvis, 2014), where agents associated with the system may have been developed by third parties, have the freedom to enter and exit the system at will, and may have goals perhaps conflicting with the objectives of the system of which they are part.

In MAS, the fundamental entity is the agent. This agent represents an element endowed with the knowledge necessary to perform some task autonomously. It can, for example, monitor certain activities performed by users of the system and interfere with assisting in the execution of tasks, or even representing the user in performing some of them. The agents will then read certain environment using sensors, process these readings taking in consideration its internal state, make decisions on how to act, and act on the environment through actuators (Wooldridge, 2009).

Agents can act alone or together with other agents, they hold their own goals and act «rationally» in the sense of accomplishing them. When communicating with other agents, they should use a communication language, and use social skills to negotiate with other agents to achieve their goals, even though sometimes those goals are not entirely aligned with the goals of the system. Moreover, it is at this moment that the need to create mechanisms that coordinate the agents is verified, so that the system functions in an organised way, or remains stable.

The Organizational Infrastructure (Weiss, 2013), (Wooldridge, 2009), bring this coordination layer for MAS. The definition of roles to be played by the agents in the system, together with norms dictating what these roles should/can do in the MAS, helps the system to have the agents performing their tasks in a coordinated way. In this sense, the autonomy of the agents can be managed in a way that, although they have their objectives, they verify that the system also has its objectives that must be observed at the time of its decision-making.

Enyo Gonçalves, Mariela Cortés, Marcos de Oliveira,

Nécio Veras, Mário Falcão and Jaelson Castro

An Analysis of Software Agents, Environ-ments

and Applications School (WESAAC): Retrospective,

Relevance, and Trends
ADCAIJ: Advances in Distributed Computing and Articial Intelligence Journal Regular Issue, Vol. 6 N. 2 (2017), 19-32 elSSN: 2255-2863 - http://adcaij.usal.es (c) Ediciones Universidad de Salamanca - CC BY 
The identification of the users' activities in the environment, the contextual information observed by the agents and the request for services between them, generate inputs for the artificial agents that are part of the MAS, generating perceptions and determining the agents' internal plans of action. These plans implement the decision-making of agents and are interpreted by internal architectures such as BDI (Beliefs, Desires and Intensions) (Wooldridge, 2009), determining when and how to interact with other elements of the system and change its state.

\section{Retrospective of the Event}

WESAAC provides a forum for researchers and a school for students. Initially called «Workshop - School of Agent Systems for Collaborative Environments» and then from the fourth edition, following suggestions from the participants of the first three editions, the event's scope was expanded without losing its trademark (acronym), and the interest that involved the researchers originally. In the fifth edition, the national scope of the event was maintained and strengthened, with the participation of prominent researchers from the MAS field, from several institutions from Brazil, such as USP, IME / RJ, UFRGS, FURG, UFSC, UCPel, UTFPR, and abroad, notably the University of Bologna (Italy). They presented lectures and led workshops on their research topics.

The first edition of WESAAC occurred in 2007 in the city of Pelotas- RS, at the Catholic University of Pelotas (UCPel), and it was coordinated by Prof. Carlos Antonio da Rocha Costa. His second edition was in 2008 in the city of Santa Cruz do Sul-RS, at the University of Santa Cruz do Sul (UNISC), and it was coordinated by Prof. Rejane Frozza. The third edition happened in 2009 in the city of Caxias do Sul-RS, at the University of Caxias do Sul (UCS), under the coordination of Prof. João Luis Tavares da Silva. The fourth edition took place in 2010 in the city of Rio Grande-RS, at the Federal University of Rio Grande (FURG), under the overall coordination of Prof. Diana Francisca Adamatti. The fifth edition took place in 2011, in Curitiba-PR, at the Federal Technological University of Paraná (UTFPR), under the overall coordination of Prof. Gustavo Alberto Giménez Lugo.

The sixth edition took place in 2012 in the city of Florianópolis-SC, at the Federal University of Santa Catarina (UFSC), under the coordination of Prof. Jomi Fred Hübner. The seventh edition happened in the city of São Paulo-SP at the University of São Paulo (USP) in 2013, under the coordination of Prof. Anarosa Alves Franco Brandão. The eighth edition occurred in Porto Alegre-RS at the Pontifical Catholic University of Rio Grande do Sul (PUCRS) in 2014, under the organisation of Prof. Rafael Bordini. The ninth and last edition took place in the city of Niterói-RJ in 2015, under the coordination of Prof. Viviane Torres da Silva.

All the proceedings and links to the home pages of WESAAC editions and the results of this research are available on a web page created by this work in: http://intranet.tiangua.ifce.edu.br/necio/wesaac-adcaij/site/.

\section{Research Method}

The experimental software engineering community has proposed reliable processes, guidelines and templates for conducting Systematic Literature Reviews (SLR) (Kitchenham and Charters, 2007). In this paper, we adopted the research strategy described in (Oliveira et al., 2013); hence, we conducted a scoping study in order to «map out» the WESAAC previous editions.

While a systematic review is a means of identifying, evaluating and interpreting the available research findings related to a research question, topic area, or phenomenon (Kitchenham and Charters, 2007). A scoping study is rather focused on examining the extent, range and nature of research activity, providing an overview of a specific area (Oliveira et al., 2013).

The set of steps applied in our paper were: (i) Protocol definition; ii) Research Questions definition; (iii) Conduction of Search; (iv) Data Extraction and Mapping; and (v) Data Analysis and Synthesis. These steps combine scoping study and systematic review good practices, such as protocol definition, to take advantage of both methodologies. The protocol has been designed and executed by four researchers with broad familiarity with MAS and one additional researcher that revised this protocol.

Enyo Gonçalves, Mariela Cortés, Marcos de Oliveira,

Nécio Veras, Mário Falcão and Jaelson Castro

An Analysis of Software Agents, Environ-ments

and Applications School (WESAAC): Retrospective,

Relevance, and Trends
ADCAIJ: Advances in Distributed Computing and Articial Intelligence Journal Regular Issue, Vol. 6 N. 2 (2017), 19-32 elSSN: 2255-2863 - http://adcaij.usal.es (c) Ediciones Universidad de Salamanca - CC BY 


\subsection{Research questions}

The research questions that we intend to answer in this scoping study are:

RQ1: What are the main authors and institutions that published in WESAAC?

RQ2: What is the main language used in the papers?

RQ3. What are the main topics discussed in WESAAC?

RQ4. What are the types of the contributions?

RQ5. What is the impact of the publications of WESAAC in the community?

RQ6. What are the trends in MAS?

\subsection{Search Strategy, Data Sources and Study Selection}

A manual search was conducted in all WESAAC proceedings to collect the studies, by examining the studies title and abstract. The following inclusion and exclusion criteria were used:

- Inclusion Criteria: All research papers published in the WESAAC;

- Exclusion Criteria: Short papers published in WESAAC.

The inclusion and exclusion criteria over the ten editions of WESAAC proceedings resulted in 128 studies to be further analysed and classified.

\subsection{Data extraction and synthesis}

After the search and the selection processes, we performed a data extraction process by analysing the 128 selected papers. To guide this data extraction, we used a predefined extraction form containing the following fields:

Identifier, Publication Year, Title, Language, Institution, Authors, Topic discussed, Type of contribution (model, tool, process, approach, method, among others) and Number of citations by type (conference, journal, book, dissertation, thesis, other).

This form enabled us to record full details of the papers under review and to be specific about how each of them addressed our research questions. The selection process and the data extraction were performed using a spreadsheet tool. In the next section, we present the results obtained in this scoping study.

\section{Results}

In this section, each research question is answered by analysing different points of views, and highlighting evidence gathered from the data extraction process.

\section{RQ1. What are the main authors and institutions that published in WESAAC?}

In these ten editions, the WESAAC had the participation of 52 institutions and 257 authors. From a total of 257 authors who published a paper at WESAAC editions, Antônio Carlos da Rocha Costa and Graçaliz Pereira Dimuro are leading the list with 18 published papers, followed by Diana Francisca Adamatti with 15 papers published. Rejane Frozza is in third place with 8 papers published. In fifth place with 5 papers published we identified four authors: Esteban de Manuel Jerez, Glenda Dimuro, Jomi Fred Hübner, Mariela Inés Cortés.

The list of the top four WESAAC authors is shown in Table 1. It is important to mention that the data illustrates the number of times in which an author is authoring or co-authoring an article.

Enyo Gonçalves, Mariela Cortés, Marcos de Oliveira,

Nécio Veras, Mário Falcão and Jaelson Castro

An Analysis of Software Agents, Environ-ments

and Applications School (WESAAC): Retrospective,

Relevance, and Trends
ADCAIJ: Advances in Distributed Computing and Articial Intelligence Journal Regular Issue, Vol. 6 N. 2 (2017), 19-32 elSSN: 2255-2863 - http://adcaij.usal.es 
Table 1: Authors with more papers published in WESAAC

\begin{tabular}{|c|c|c|c|}
\hline Placement & Author & Institution & \#papers \\
\hline \multirow{2}{*}{1} & Antônio Carlos da Rocha Costa & $\begin{array}{c}\text { Universidade Federal do Rio Grande do Sul } \\
\text { (UFRGS)/Universidade Federal do Rio Grande } \\
\text { (FURG) }\end{array}$ & 18 \\
\cline { 2 - 4 } & Graçaliz Pereira Dimuro & Universidade Federal do Rio Grande (FURG) & 18 \\
\hline \multirow{2}{*}{2} & Diana Francisca Adamatti & Universidade Federal do Rio Grande (FURG) & 15 \\
\hline \multirow{2}{*}{3} & Rejane Frozza & Universidade de Santa Cruz do Sul (UNISC) & 8 \\
\cline { 2 - 5 } & Esteban de Mauel Jerez & Universidad de Sevilla & 5 \\
\cline { 2 - 5 } & Glenda Dimuro & Universidad de Sevilla & 5 \\
\cline { 2 - 5 } & Jomi Fred Hübner & Universidade Federal de Santa Catarina (UFSC) & 5 \\
\cline { 2 - 5 } & Mariela Inés Cortés & Universidade Estadual do Ceará (UECE) & 5 \\
\hline
\end{tabular}

As it has already been mentioned, 45 institutions have had at least one publication at WESAAC. See in Table 2 the top five institutions with the most number of publications.

Table 2: The number of papers of the top 6 (six) institutions

\begin{tabular}{|c|c|c|}
\hline Placement & Institution & \# papers \\
\hline 1 & Universidade Federal do Rio Grande (FURG) & 24 \\
\hline 2 & Universidade Católica de Pelotas (UCEPEL) & 22 \\
\hline 3 & Universidade Federal de Santa Catarina (UFSC) & 11 \\
\cline { 2 - 3 } & Universidade Federal do Rio Grande do Sul (UFRGS) & 11 \\
\hline 4 & Universidad de Sevilla & 10 \\
\hline 5 & Universidade de Santa Cruz do Sul (UNISC) & 8 \\
\hline
\end{tabular}

Although almost all the universities listed in Table 2 are in the southern region, we can mention further ones, such as Universidade de São Paulo (7th place), Universidade Estadual do Ceará (12th place) and Universidade Federal Fluminense (13th place) located in different regions. That shows that despite the hegemony of the Southern Brazilian universities, there is the participation of other regions of the country, and foreign universities as well. WESAAC started in the south of Brazil and researchers of these regions were involved in most of the event editions. Therefore, it is natural that researchers and universities of that region are well ranked.

After the identification of the most participating authors and institutions, an analysis of the main languages and the most discussed topics was performed.

\section{RQ2: What is the main language used in the papers?}

We also investigated the language in which the papers were written, and we identified the percentage of papers written in English and Portuguese. 103 papers (80,5\%) were written in Portuguese, and 25 papers (19,5\%) were written in English. However, we can note an increase in the number of papers in English in the 2011-2016 period compared with the period of 2007-2011. Figure 1 shows the number of papers and the progress.

Enyo Gonçalves, Mariela Cortés, Marcos de Oliveira, Nécio Veras, Mário Falcão and Jaelson Castro An Analysis of Software Agents, Environ-ments and Applications School (WESAAC): Retrospective, Relevance, and Trends
ADCAIJ: Advances in Distributed Computing and Articial Intelligence Journal Regular Issue, Vol. 6 N. 2 (2017), 19-32 elSSN: 2255-2863 - http://adcaij.usal.es (c) Ediciones Universidad de Salamanca - cC BY 

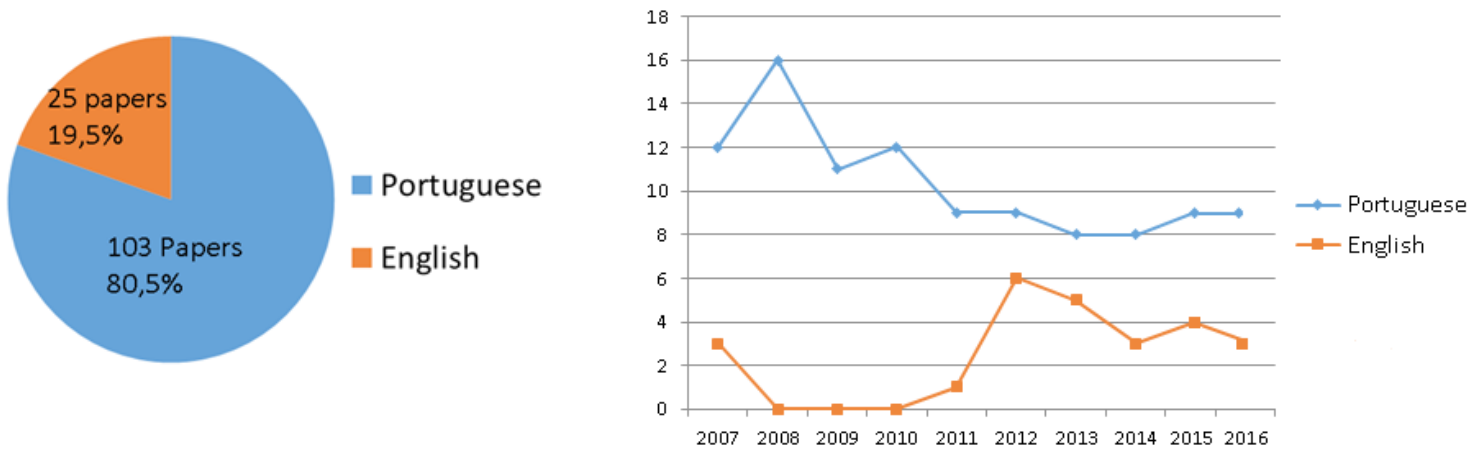

Figure 1: The progress of language used in WESAAC papers

\section{RQ3. What are the main topics discussed in WESAAC?}

All papers were analysed to evaluate MAS topics that they have addressed. The list of discussed topics was based on the call for papers of previous editions of the WESAAC. Each paper was classified in one the topics. The main topics defined according to the papers are shown in Table 3. "Applications of agents and multi-agent systems» is the main discussed topic $(39.8 \% ; 51$ papers), followed by «agent-based software development (programming languages, platforms, tools, methodologies)» (16.4\%; 21 papers). «Social simulations and agentbased simulation» follows closely with $13.3 \%$ (17 papers).

Table 3: The discussed topics in WESAAC

\begin{tabular}{|c|c|c|}
\hline Topic & \#papers & \% \\
\hline applications of agents and multi-agent systems & 51 & 39,8 \\
\hline agent-based software development & 21 & 16,4 \\
\hline social simulations and agent-based simulation & 17 & 13,3 \\
\hline cooperation/coordination (negotiation, argumentation, reputation) & 13 & 10,1 \\
\hline agent organisations, societal issues, normative systems & 9 & 7,0 \\
\hline agent architectures and theories (BDI, belief revision, automated reasoning) & 8 & 6,2 \\
\hline (formal) specification and verification of multi-agent systems & 5 & 4 \\
\hline agent communication & 4 & 3,2 \\
\hline
\end{tabular}

\section{RQ4. What are the types of the contributions?}

The purpose of this research question was to identify the contributions types of the WESAAC papers. The results of this question are presented in Table 4.

Enyo Gonçalves, Mariela Cortés, Marcos de Oliveira, Nécio Veras, Mário Falcão and Jaelson Castro An Analysis of Software Agents, Environ-ments and Applications School (WESAAC): Retrospective, Relevance, and Trends
ADCAIJ: Advances in Distributed Computing and Articial Intelligence Journal Regular Issue, Vol. 6 N. 2 (2017), 19-32 eISSN: 2255-2863 - http://adcaij.usal.es (c) Ediciones Universidad de Salamanca - CC BY 
Table 4: WESAAC papers types of contributions

\begin{tabular}{|c|c|c|}
\hline Type of Contribution & \#papers & $\mathbf{\%}$ \\
\hline A Multiagent System implementation & 28 & 21,9 \\
\hline Architecture/Framework & 22 & 17,2 \\
\hline Tool & 20 & 15,7 \\
\hline AI model/Formal model/Mathematical model/Ontology & 17 & 13,3 \\
\hline Simulation & 8 & 6,2 \\
\hline A multiagent Technique & 6 & 4,7 \\
\hline AI Technique implementation & 5 & 3,9 \\
\hline Algorithm & 5 & 3,9 \\
\hline Methodology & 5 & 3,9 \\
\hline Evaluation & 5 & 3,9 \\
\hline Multiagent System modelling & 2 & 2,2 \\
\hline Programming Language & & 1,6 \\
\hline Discussion or Review & 2 & 1,6 \\
\hline
\end{tabular}

We tried to list the types according to the classification presented in the papers, i.e. Simulation, Illustration, Experiment, Comparison and Survey. Papers without validation were classified as No Validation. Note that, similarly to other research questions, this question also allows a study to be included in only one category. In Table 4 the predominant contribution that we identified was «A Multiagent System implementation» $(21.9 \% ; 28$ studies), followed by «Architecture/Framework» (17.2\%; 22 studies) and «Tool» (15.7\%; 20 studies).

The topic «AI model/Formal model/Mathematical model/Ontology» is proposed by more than $13,79 \%$ of the papers. The «Simulation» topic is suggested by more than $6,89 \%$ of the papers.

We also investigated if the contributions were empirically validated. Figure 3 shows that «simulation» is the main type of validation (23.3\%, 30 papers), followed by «illustration» $(18.6 \%, 24$ papers). The validation «experiment» was performed by $16.3 \%$ (21 papers), «comparison» was conducted by $10,8 \%$ (14 papers). $1.5 \%$ ( 2 papers) performed «survey». Some papers did not present any validation $(29.5 \%, 38$ papers).

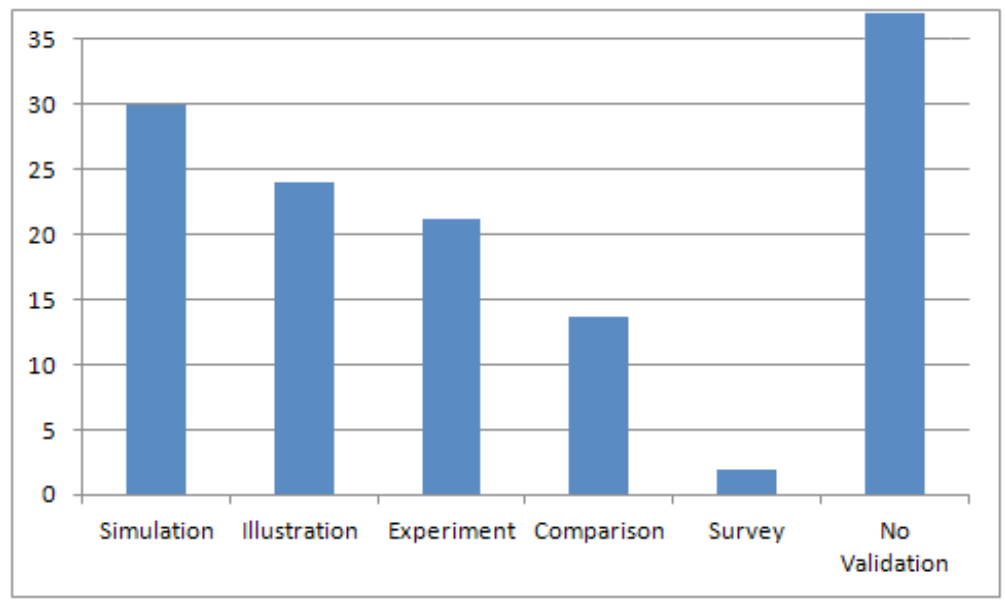

Figure 3: Empirical studies

Enyo Gonçalves, Mariela Cortés, Marcos de Oliveira, Nécio Veras, Mário Falcão and Jaelson Castro An Analysis of Software Agents, Environ-ments and Applications School (WESAAC): Retrospective, Relevance, and Trends
ADCAIJ: Advances in Distributed Computing and Articial Intelligence Journal Regular Issue, Vol. 6 N. 2 (2017), 19-32 eISSN: 2255-2863 - http://adcaij.usal.es (c) Ediciones Universidad de Salamanca - CC BY 


\section{Q5. What is the impact of the publications of WESAAC in the community?}

A deep investigation over the WESAAC papers shows the numbers of WESAAC referred papers over the years. This investigation was conducted by manually analysing all citations of each 128 papers through Google Scholar.

Regarding the publication venues of the papers that cite WESAAC papers, we found the following distribution (see Figure 2): 51\% in conferences (28 studies, which 9 studies in international conferences and 5 studies in WESAAC editions); $5 \%$ in journals (3 studies, which 2 studies in international journals); $2 \%$ in PhD Thesis (1 study), 29\% in Master Thesis (16 studies, which 1 international citation) and 13\% (7 studies) in others types of documents (technical reports, presentations, among others).

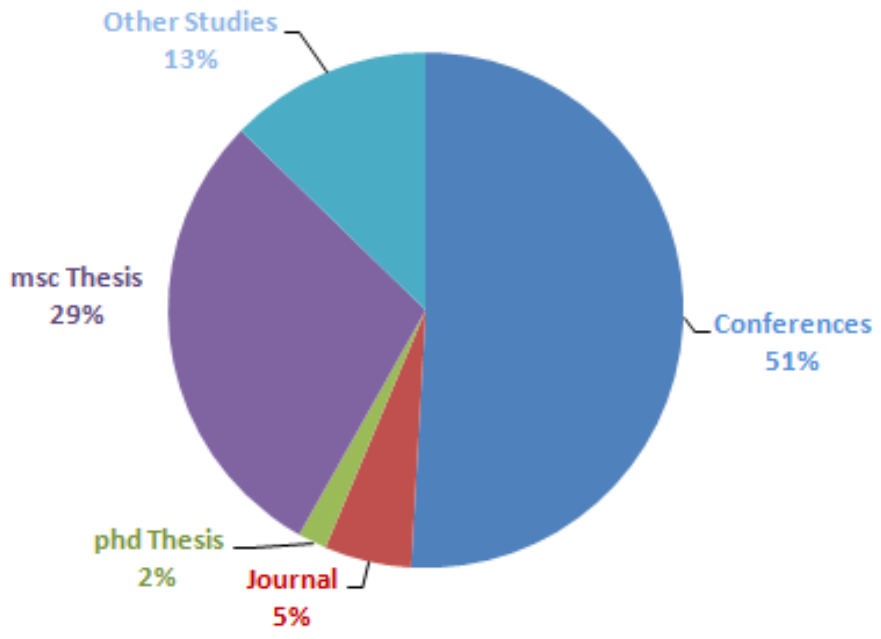

Figure 2: Publication venues of WESAAC papers

The top 5 most cited papers are listed in Table 5 . These papers represent the papers from the WESAAC that had the greatest research impact, considering citation count.

Table 5: TOP 5 WESAAC most cited papers

\begin{tabular}{|c|c|c|c|}
\hline Paper & Authors & Year & Citations \\
\hline $\begin{array}{c}\text { Agentes tutor e companheiro em um ambiente } \\
\text { educacional baseado em estilos cognitivos }\end{array}$ & $\begin{array}{c}\text { Rejane Frozza, Andréa Konzen, Alessandra } \\
\text { G. Mainieri, Jacques Schreiber, Kurt Molz, } \\
\text { Jorge Tautz, Ricardo Pedó, Jonas Dresch }\end{array}$ & 2007 & 6 \\
\hline $\begin{array}{c}\text { Modelando a Organização Social de um SMA para } \\
\text { Simulacão dos Processos de Produção e Gestão } \\
\begin{array}{c}\text { Social de um Ecossistema Urbano: o caso da Horta } \\
\text { San Jerónimo da cidade de Sevilla, Espanha }\end{array}\end{array}$ & $\begin{array}{c}\text { Flávia C. P. Santos, Glenda Dimuro, Thiago } \\
\text { F. Rodrigues, Diana F. Adamatil, Graçaliz } \\
\text { P. Dimuro, Antônio C. R. Costa, Esteban de } \\
\text { Manuel Jerez }\end{array}$ & 2012 & 5 \\
\hline $\begin{array}{c}\text { Simulação Multiagente de uma Abordagem } \\
\text { Evolutiva e Espacial para o Jogo do Ultimato }\end{array}$ & $\begin{array}{c}\text { Luís Felipe K. Macedo, Murian dos R. Ribeiro, } \\
\text { Stephanie L. Brião, Celso N. da Fonseca, } \\
\text { Marilton S. de Aguiar, Graçaliz P. Dimuro }\end{array}$ & 2012 & 4 \\
\hline $\begin{array}{c}\text { Fred - um agente pedagógico mediador na } \\
\text { construção do conhecimento }\end{array}$ & $\begin{array}{c}\text { Julio Cezar Souza de Mello, Rejane Frozza } \\
\text { Uma Arquitetura de Referência para Softwares }\end{array}$ & 2007 & 4 \\
\hline $\begin{array}{c}\text { Assistentes Pessoais Baseada em Agentes e SOA } \\
\text { Saulo Popov Zambiasi, Ricardo J. Rabelo }\end{array}$ & 2011 & 4 \\
\hline
\end{tabular}

Enyo Gonçalves, Mariela Cortés, Marcos de Oliveira, Nécio Veras, Mário Falcão and Jaelson Castro An Analysis of Software Agents, Environ-ments and Applications School (WESAAC): Retrospective, Relevance, and Trends
ADCAIJ: Advances in Distributed Computing and Articial Intelligence Journal Regular Issue, Vol. 6 N. 2 (2017), 19-32 elSSN: 2255-2863 - http://adcaij.usal.es (c) Ediciones Universidad de Salamanca - CC BY 
The most cited paper is «Agentes tutor e companheiro em um ambiente educacional baseado em estilos cognitivos», published in 2007. It was cited 6 times and authored by Rejane Frozza, Andréa Konzen, Alessandra G. Mainieri, Jacques Schreiber, Kurt Molz, Jorge Tautz, Ricardo Pedó, Jonas Dresch.

The topics of the most cited paper are in accordance with the main topics discussed in WESAAC (RQ3). The first-place paper addresses the «A Multiagent System implementation». The second most quoted paper addresses the topic «agent organisations, societal issues, normative systems». While the first work in third place, from 2012, is from "social simulations and agent-based simulation». Finally, the other papers tied in third place cover «applications of agents and multi-agent systems» and «agent architectures and theories (BDI, belief revision, automated reasoning)».

Regarding empirical studies, «illustration» was used to validate the paper in first place and the second place. The third-place papers used «comparison», not presented a validation and «simulation», respectively.

We also analysed which conferences have most referenced WESAAC papers. Regarding conference papers, Table 6 shows the conferences that most cite WESAAC papers respectively.

Table 6: TOP 5 conferences

\begin{tabular}{|c|c|}
\hline Conference & \#citations \\
\hline Software Agents, Environments and Applications School (WESAAC) & 5 \\
\hline Brazilian Workshop on Social Simulation & 4 \\
\hline Latin American Informatics Conference (CLEI) & 2 \\
\hline
\end{tabular}

Based on these results, it is possible to see that the papers are serving as good sources to define and evolve the already proposed techniques, methods, processes and so on. However, the references from WESAAC papers itself is considered low and could be encouraged in the future call for papers. Low amount of citations in journal papers and international conferences should be mentioned, and probably it is related to few articles written in English (22 papers). Extended versions of the best papers could be published in a journal, as other events have been doing, and this could encourage more authors to submit. The 55 articles that cite WESAAC papers involve the participation of 15 researchers from other countries (Not Brazil) and 62 researchers that have not previously published papers at WESAAC.

\section{RQ6. What are the trends in MAS?}

The purpose of this question is to investigate the trends in MAS in relation to the topics addressed by the WESAAC papers and other papers of the MAS area. In this case, we consider the main topics defined according to the papers showed in Table 3, i.e., the top 5 topics along the ten editions, and some papers related to our results.

Figure 3 shows the number of publications per topic/year. According to this figure, it is possible to observe an increase in the following topics: Agent-based software development and Agent organisations, societal issues, normative systems. On the other hand, we have observed a decrease in the number of papers on the following: applications of agents and multi-agent systems and social simulations and agent-based simulation. The cooperation/coordination topic remained without significant variations.

Enyo Gonçalves, Mariela Cortés, Marcos de Oliveira, Nécio Veras, Mário Falcão and Jaelson Castro An Analysis of Software Agents, Environ-ments and Applications School (WESAAC): Retrospective, Relevance, and Trends
ADCAIJ: Advances in Distributed Computing and Articial Intelligence Journal Regular Issue, Vol. 6 N. 2 (2017), 19-32 elSSN: 2255-2863 - http://adcaij.usal.es (c) Ediciones Universidad de Salamanca - CC BY 


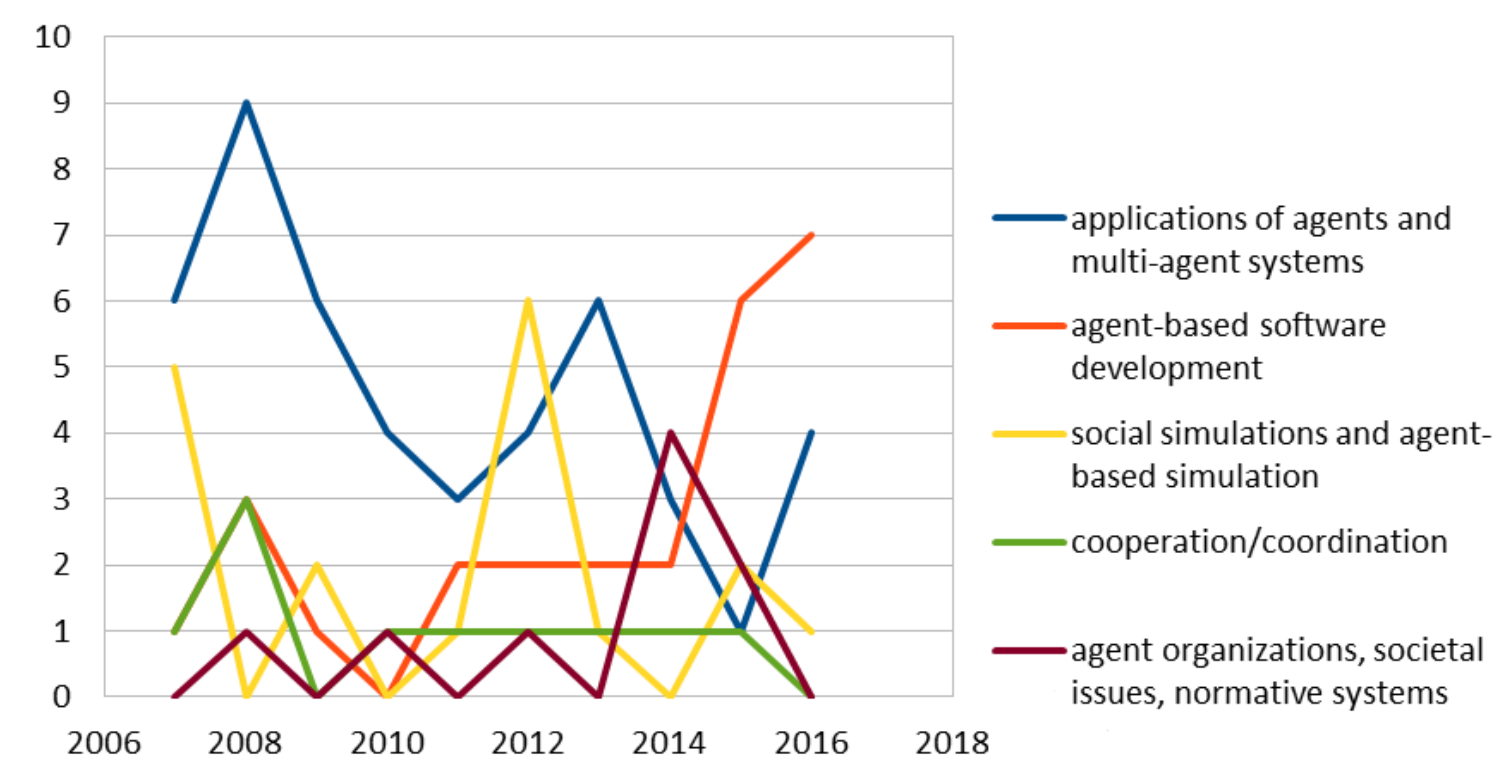

Figure 3: Number of WESAAC publications per topic/year

Figure 4 shows the number of publications per contribution/year, where we can note that all contributions have variations across the editions. According to this figure, it is possible to observe an increase in the last edition for the following contributions: «A Multiagent system implementation» and «Simulation». On the other hand, it has been noted a decrease in the number of papers related to «Architecture/Framework». The «Tool» contribution has a variation, and it was not proposed in the last two editions of WESAAC. The «AI model/Formal model/Mathematical model/Ontology» contribution remained without significant variations.

The peak of «Tool» contribution was in 2012 and «architecture/framework» was in 2010, with 5 papers each one. The topmost of «A Multiagent System implementation» was in 2008 with 6 papers. The top of «Simulation» was in 2012 with 3 papers and «AI model/Formal model/Mathematical model/Ontology» was in 2016 with 5 papers.

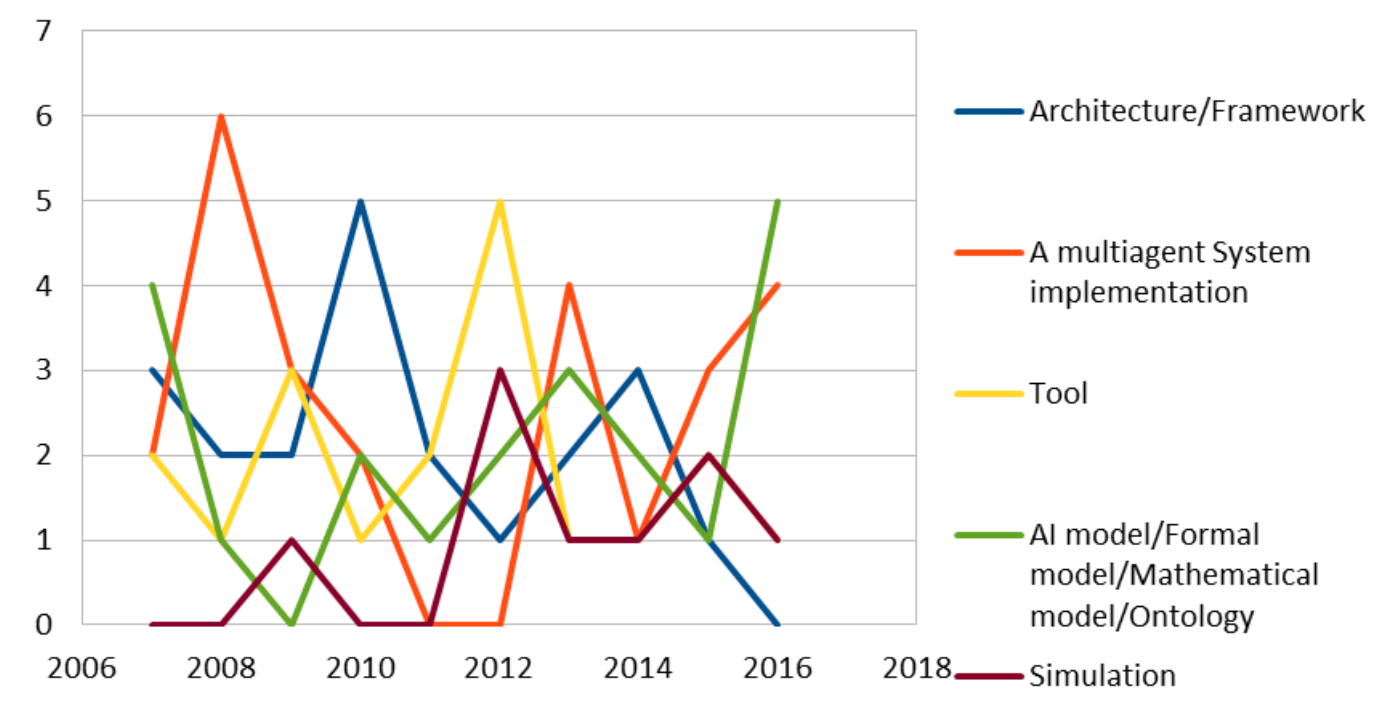

Figure 4: Number of WESAAC publications per contribution/year

Enyo Gonçalves, Mariela Cortés, Marcos de Oliveira, Nécio Veras, Mário Falcão and Jaelson Castro An Analysis of Software Agents, Environ-ments and Applications School (WESAAC): Retrospective, Relevance, and Trends
ADCAIJ: Advances in Distributed Computing and Articial Intelligence Journal Regular Issue, Vol. 6 N. 2 (2017), 19-32 eISSN: 2255-2863 - http://adcaij.usal.es (c) Ediciones Universidad de Salamanca - CC BY 
Since 2005 specific technical challenges of agent-based computing are discussed: Trust and reputation, Virtual organisation formation and management, Resource allocation and coordination, Negotiation, Emergence in large-scale agent systems, Learning and optimisation theory, Methodologies, Provenance, Service architecture and composition, and Semantic integration (Luck et al., 2005).

In that roadmap the authors built fifteen recommendations about challenges appointed, and some can be found in the history of WESAAC, such as: «Creation of tools, techniques and methodologies to support agent systems developers», «Develop techniques for expressing and reasoning about trust and reputation, on both individual and social level, to enable interaction in dynamic and open environments», and "Integrate agent components and features to enable the different theories, technologies and infrastructures to come together coherently».

On other hand, recommendations such as «Develop a range of new techniques for learning and optimization in dynamic and unstable multi-agent environments, together with evaluation methods» and «Establish appropriate trade-offs between adaptability and predictability so that agents can exhibit behaviour, emergent or otherwise, that can be supported by tools and property verification» were not exploited by authors in any paper.

According to (Sturm et al., 2014), Agent-Oriented Software Engineering can be divided into the following themes: Agent Programming Languages, Agent Oriented Frameworks, Agent Oriented Architectures, Agent Oriented Methodologies (Modelling Techniques) and Agent Communication (Content Languages, Interaction Mechanisms). The research community in the last almost 20 years, has explored these themes and the Applications built using technologies that come from them.

In (Dix et al., 2012) is made a report on the outcomes of the discussions of several researchers in the field, from universities and industry. They were from distinct areas and were engaged on work related to the following subjects: avionics and defence, smart cities, transportation, logistics, workflow management, decision support, healthcare, personal assistants, real-time control systems, power engineering, information integration, and virtual environments. They have concentrated the discussion on the following themes: Integration of MAS with other systems and technologies, and validation of MAS; Coordination and Organisation; Tool, Languages and Technologies; and Component-Oriented Agent Design. In those surveys (Dix et al., 2012), the major concern was to drive efforts from the research community to find out why agent technologies were not embraced yet by the industry, and what is necessary to make that happen. They have pointed out some strategies to that end, such as: concentrate on making better tools and not only building new ones; create ways to validate and test MAS for safety, security, scalability, quality, maintainability, performance and interoperability, for example; componentize agent technology to better integrate it with other types of systems, concentrate on the scalability of the organizations and the runtime environments to support that. There is a concern as well to measure how the Agent Oriented techniques compare against each other, and with other technologies.

\section{Threats to Validity}

There are some threats to the validity of our study, which we briefly describe along with the mitigation strategy for them.

In order to analyse the top authors, institutions, and countries, the number of papers for each one was considered. During this process, no distinction was made regarding the co-authoring of studies. The main author receives the same score as co-authors. We also should consider that authors change of intuitions and countries. As the analysis of the citations of each 128 papers was performed manually, we considered it as an error prone activity. To mitigate this issue, some classifications were double checked.

Finally, we could have explored a broader set of data to investigate other aspects of the papers included in the review. Moreover, it is possible that some kind of inaccuracy or misclassification may have occurred in the data extraction performed in this scoping study.

Enyo Gonçalves, Mariela Cortés, Marcos de Oliveira,

Nécio Veras, Mário Falcão and Jaelson Castro

An Analysis of Software Agents, Environ-ments

and Applications School (WESAAC): Retrospective,

Relevance, and Trends
ADCAIJ: Advances in Distributed Computing and Articial Intelligence Journal Regular Issue, Vol. 6 N. 2 (2017), 19-32 elSSN: 2255-2863 - http://adcaij.usal.es (c) Ediciones Universidad de Salamanca - CC BY 


\section{Related Works}

The analysis of how the Software Engineering (SE) area as well as the Requirements Engineering is evolving has been the topic of some studies (Dermeval et al., 2015), (Oliveira et al., 2011), (Laski et al., 2014), (Juziuk, Weyns and Holvoet, 2014) and (Isern and Moreno, 2016).

In (Dermeval et al.,2015), the authors investigate about how ontologies support RE and identify to what extent they have been applied to this field. They conducted a SLR to identify the primary studies on the use of ontologies in RE, following a predefined review protocol. They identified the main RE phases addressed, the requirements modelling styles that have been used in conjunction with ontologies, the types of requirements that have been supported using ontologies and the ontology languages that have been adopted. Finally, the authors examined the types of contributions reported and looked for evidences of the benefits of ontology-driven RE.

A scoping study was conducted by (Oliveira et al., 2011) in which the authors analysed 512 papers of the 24 editions of Brazilian Symposium on Software Engineering (SBES) and understanding which is the impact of international research in this event. Their findings suggest that greater attention should be given to the SE area, with the aim to attract research from industry with real data, and international collaboration. The celebration of 25th anniversary of the SBES, as well as the realisation of the Requirements Engineering Conference in Brazil for the first time, motivated (Oliveira et al., 2011) to conduct a mapping study aiming to have a closer look at the local RE community. Their results showed that the Brazilian researchers had been extensively publishing at SBES and WER. Moreover, their findings reveal the better empirical validation may be required.

A revision of 258 papers published at WER was performed by (Laski et al., 2014). Their results are in a certain way like ours: Brazil is one of the countries with the most number of published papers in RE. Moreover, Universidade Federal de Pernambuco (UFPE) is one of the most participating institutions as far as publications at WER are concerned.

There are some Systematic Literature Reviews in about MAS. In (Juziuk, Weyns and Holvoet, 2014), the authors performed a SLR covering the main publication venues of the field since 1998, resulting in 206 patterns. The study shows that (i) there is a lack of a standard template for documenting design patterns for MAS, which hampers the use of patterns by practitioners, (ii) associations between patterns are poorly described, which results in a lack of overview of the pattern space, (iii) patterns for MAS have been used for a variety of application domains, which underpins their high potential for practitioners, and (iv) classifications of design patterns for MAS are bounded to specific pattern catalogues, a more holistic view of the pattern space is missing..

The purpose of the review presented in (Isern and Moreno, 2016) is to show the feasibility of applying intelligent agents in the healthcare domain and use the findings to provide a discussion of current trends and devise future research directions. The authors present a review of the most recent literature (2009-2014) of applications of agents in healthcare is discussed, and two classifications considering the main goal of the health systems as well as the main actors involved have been investigated.

\section{Conclusions and Future Works}

In order to understand how the MAS community evolves, this study presented some findings resulted from a scoping study considering the ten editions of WESAAC. In this sense, we identified the most active countries, institutions and authors, the main topics discussed, the types of the contributions, the conferences and journals that have most referenced WESAAC papers, the publications with the greatest impact, and trends in MAS.

We identified the top 13 authors, which predominantly are from south of Brazil. We also recognised a tendency of increase in papers written in English in last five years. We also presented eight topics discussed in papers and a number of papers classified in each of them. Thirteen categories for type of contribution were used to classify the papers. We also analysed the means used for validation, i.e. Simulation, Illustration, Experiment, Comparison, Survey and No validation. We also checked the impact of WESAAC papers in main venues as journal papers, conference papers, master thesis, doctoral thesis and others. Finally, we analysed some trends related to trends WESAAC papers related to MAS.

Enyo Gonçalves, Mariela Cortés, Marcos de Oliveira,

Nécio Veras, Mário Falcão and Jaelson Castro

An Analysis of Software Agents, Environ-ments

and Applications School (WESAAC): Retrospective,

Relevance, and Trends
ADCAIJ: Advances in Distributed Computing and Articial Intelligence Journal Regular Issue, Vol. 6 N. 2 (2017), 19-32 elSSN: 2255-2863 - http://adcaij.usal.es (c) Ediciones Universidad de Salamanca - CC BY 
Only 19,5\% of published papers were written in English. One way to encourage submission of articles in English is the publication of articles selected from the event in an international journal, and this action was taken on WESAAC 2016. In this way, we believe that more publications in English and the possibility of publication in a journal can positively influence the impact of WESAAC's works. Stimulating the citation of WESAAC papers in new submissions may increase the access and reading of WESAAC papers and consequently improving their impact through citations not only in WESAAC and other events too.

Future works include an analysis to discuss the schools (e.g. for their suitability, themes, repetition, impact on participants' dissertations/thesis or papers). An interview with experts in the area can also be a good way to understand the conference trends along the years (Oliveira et al., 2011).

\section{Acknowledgments}

This work was supported by Conselho Nacional de Desenvolvimento Científico e Tecnológico (CNPq) - Brazil.

\section{References}

10th Software Agents, Environments and Applications School (WESAAC) Available at: http://intelligentagents.github.io/wesaac-2016/en/.

9th Software Agents, Environments and Applications School (WESAAC) Available at: http://www.inf.pucrs.br/ felipe.meneguzzi/wesaac2014/history.html.

De Oliveira, M., Gonçalves, E., Purvis, M. Institutional Environments: A Framework for the Development of Open Multiagent Systems. Ibero-American Conference on Artificial Intelligence. Springer International Publishing, 2014.

Dix, Jürgen, Hindriks, K., Logan, B. Wayne Engineering multi-agent systems (Dagstuhl seminar 12342). Dagstuhl Reports 2.8, 2012, Available in http://drops.dagstuhl.de/opus/volltexte/2012/3784/.

Dermeval, D., Vilela, J., Bittencourt, I., Castro, J., Isotani, S., Brito, P., Silva, A. Applications of ontologies in requirements engineering: a systematic review of the literature. In: Requirements Engineering Journal, 2015.

Oliveira, K., Pimentel, J., Santos, E., Demerval, D., Gedes, G., Souza, C., Soares, M., Castro, J., Alencar, F., Silva, C . 25 years of Requirements Engineering in Brazil: a systematic mapping. In: 16th Requirements Engineering Workshop (WER 2013), 2013, Montevideo. Proceedings of the 16th Requirements Engineering Workshop - WER 2013, 2013. pages 1-17.

Isern, D., Moreno, A. A systematic literature review of agents applied in healthcare. Journal of medical systems 40.2 (2016): 43.

Laski, J.; Stancke, W.; Reinehr, S.; Malucelli, A. WER Overview: Retrospective, Trends and Relevance. In: CLEI Electronic Journal, vol. 17, n. 3, pages 4-4, 2014.

Kitchenham, B., Charters, S. Guidelines for performing systematic literature reviews in software engineering. In: Technical report EBSE 2007-001, Keele University and Durham University Joint Report, 2007.

Petersen, K., Feldt, R., Mujtaba, S., Mattsson, M. Systematic mapping studies in software engineering. In: EASE '08: Proceedings of the 12th International Conference on Evaluation and Assessment in Software Engineering, University of Bari, Italy, 2008.

Jennings, N. R. Coordination Techniques for Distributed Artificial Intelligence. In: Foundations of Distributed Artificial Intelligence, 1996, pages 187-210, Wiley.

Juziuk, J., Weyns, D., Holvoet, T. Design patterns for multi-agent systems: a systematic literature Review. Agent-Oriented Software Engineering. Springer Berlin Heidelberg, 2014.

Luck, M., McBurney, P., Shehory, O., Willmott, S. Agent technology: computing as interaction (a roadmap for agent based computing), University of Southampton on behalf of AgentLink III, 2005.

Russell, S., Norvig, P. Artificial Intelligence: A Modern Approach, 2nd Ed., 2003, Upper Saddle River, NJ: Prentice Hall, ISBN 0-13-790395-2.

Enyo Gonçalves, Mariela Cortés, Marcos de Oliveira,

Nécio Veras, Mário Falcão and Jaelson Castro

An Analysis of Software Agents, Environ-ments

and Applications School (WESAAC): Retrospective,

Relevance, and Trends
ADCAIJ: Advances in Distributed Computing and Articial Intelligence Journal Regular Issue, Vol. 6 N. 2 (2017), 19-32 elSSN: 2255-2863 - http://adcaij.usal.es (c) Ediciones Universidad de Salamanca - CC BY 
Weiss, G. Multiagent Systems (Intelligent Robotics and Autonomous Agents series)» (2013).

Wooldridge, M. An Introduction to Multiagent Systems. Second Edition. Willey, 2009.

Sturm, A., Shehory, O. Agent-Oriented Software Engineering: Revisiting the State of the Art. Agent-Oriented Software Engineering. Springer Berlin Heidelberg, 2014.

Zambonelli, F., Jennings, N., Wooldridge, M. Organizational Abstractions For The Analysis And Design Of Multi-Agent Systems. In: Agent-Oriented Software Engineering, LNCS 1957, Berlin: Springer, 2001, pages $127-141$.

Enyo Gonçalves, Mariela Cortés, Marcos de Oliveira,

Nécio Veras, Mário Falcão and Jaelson Castro

An Analysis of Software Agents, Environ-ments

and Applications School (WESAAC): Retrospective,

Relevance, and Trends
ADCAIJ: Advances in Distributed Computing and Articial Intelligence Journal Regular Issue, Vol. 6 N. 2 (2017), 19-32 elSSN: 2255-2863 - http://adcaij.usal.es (c) Ediciones Universidad de Salamanca - CC BY 